Centro de Estudios Avanzados. Departamento de Nutrición. Facultad de Ciencias de la Salud. Universidad de Playa Ancha, Valparaíso, Chile.

a Nutricionista. Magíster en Planificación en Alimentación y Nutrición. Magister en Nutrición Humana y Calidad de los Alimentos.

Recibido el 13 de abril de 2015 , aceptado el 14 de septiembre de 2015.

Correspondencia a: Mirta Crovetto M. Avenida Playa Ancha 890 Valparaíso, Chile.

Teléfono: 32-2500228 mirtacrovetto@gmail.com mcrovetto@upla.cl

\section{¿Es suficiente el recurso humano nutricionista en el sistema hospitalario público en Chile?: Una deuda pendiente}

\author{
MIRTA CROVETTO M. ${ }^{\mathrm{a}}$
}

\section{Adequacy of nutritionist (dietician) resources in Chilean public hospitals}

\begin{abstract}
Background: Human resource deficit is an important management problem in Chilean public hospitals. Aim: To analyze the adequacy of Nutritionist (Dietician) resources in public hospitals. Material and Methods: A questionnaire about Nutritionist resources was sent to head Nutritionists of all public Chilean hospitals, asking about the number of Nutritionists per service, number of hospital beds and number of daily rations served. Results were analyzed based on the Technical Guideline about Nutritional and Feeding Services of public hospitals issued by the Chilean Ministry of Health in 2005. Results: According to the guideline, there should be 1,396 nutritionists working in public hospitals and the results of the survey showed that there were only 603 professionals with a 57\% deficit. Conclusions: There is a huge gap between the amount of Nutritionists (Dieticians) required and those effectively working in public hospitals.
\end{abstract}

(Rev Med Chile 2015; 143: 1468-1477)

Key words: Dieticians; Health Resources; Nutrition; Public health administration.
E n Chile, para mejorar la calidad de la atención en salud en el nivel secundario, una de las variables básicas a considerar es el recurso humano mínimo requerido para dar respuesta en forma oportuna y eficiente a las necesidades de los establecimientos de salud. El Ministerio de Salud de Chile (MINSAL) propone en los objetivos sanitarios de la década el mejorar la accesibilidad, la equidad, la calidad y la eficiencia en la atención de las distintas prestaciones en salud que se realizan a la población ${ }^{1}$.

A su vez, en los "Objetivos de Salud del Milenio", la Organización Mundial de la Salud (OMS) señala que para lograr las metas en salud, es fundamental la política en recursos humanos que implementen los países en base a los énfasis en el "Llamado a la Acción de Toronto para una Década de Recursos Humanos en Salud. 2006-2015" y de la Organización Panamericana de la Salud (OPS) para lograr las "Metas Regionales de Recursos Humanos en Salud", que permitan la construcción de políticas e intervenciones en salud ${ }^{2-5}$.

En Chile, el perfil epidemiológico prevalente se caracteriza por un predominio de las enfermedades crónicas no trasmisibles (ECNT) y degenerativas como principales causas de muerte y discapacidad prematura en América Latina y el Caribe, con altos costos en salud ${ }^{6,7}$. Los estudios realizados por el MINSAL, para estimar la carga de enfermedad usando como indicador los años de vida ajustados por discapacidad (AVISA), por muerte prematura (AVPM) y los años vividos con discapacidad (AVD), en los años 1997 y 2007, señalan que dos factores principales, muerte prematura y discapacidad, requieren la implementación de programas focalizados y oportunos frente a las necesidades de atención especializada en todos los niveles en salud ${ }^{8,9}$. 
En el nivel secundario de salud, el nutricionista se relaciona disciplinariamente con las patologías de origen nutricional, por lo cual, la atención especializada en nutrición es fundamental para la recuperación del paciente. El MINSAL elaboró, en el año 2005, la norma técnica que regula la organización y el funcionamiento de los servicios de alimentación y nutrición de los establecimientos de salud y unidades centrales de producción de alimentos, con procesos científicamente planificados y técnicamente ejecutados ${ }^{10}$. Señala la dotación mínima de nutricionistas que cada recinto hospitalario debe disponer, según la complejidad de éste, que permita dar la atención especializada y de calidad (Tabla 1).

A su vez, la Resolución Exenta $N^{\circ} 209$ del MINSAL, de abril de 2005, describe el indicador número de nutricionistas por camas según servicio clínico (Medicina, Cirugía, Traumatología y otros) requeridos para la realización de las actividades técnicas definidas en la "Norma Técnica para Servicios de Alimentación y Nutrición" con el paciente hospitalizado (visita con evaluación nutricional, formulación dieto-terapéutica, estudio dietético, control de ingesta, entre otras) ${ }^{11}$ (Tabla 2).

En ese contexto, el objetivo de este estudio fue conocer la dotación del recurso nutricionista en los hospitales públicos del país respecto a las normativas ministeriales; "Norma Técnica del Servicio de Alimentación y Nutrición" (MINSAL 2005) y Resolución Exenta, abril de 2005 de Servicios Clínicos, para cuantificar la dotación, la cobertura y el déficit del recurso nutricionista en los establecimientos hospitalarios públicos.

\section{Material y Métodos}

Se realizó un estudio cuantitativo descriptivo transversal no experimental, se analizaron las variables cuantitativas, dotación de nutricionistas al año 2012 y requerimientos de nutricionistas en hospitales públicos respecto a las normativas vigentes.

El universo correspondió a la totalidad de los hospitales públicos de Chile, organizados administrativamente por regiones y servicios de salud. La muestra fue de 170 establecimientos hospitalarios públicos.

La recolección de datos fue realizada mediante la aplicación de un cuestionario enviado a cada
Tabla 1. Clasificación de los servicios de alimentación y nutrición en servicios de máxima, mediana y baja complejidad según número de raciones*

\begin{tabular}{|ll|}
\hline Clasificación & Número de raciones \\
\hline Máxima complejidad & 300 almuerzos y más \\
\hline Media complejidad & 150 a 299 almuerzos \\
\hline Mínima complejidad & menos de 150 almuerzos \\
\hline
\end{tabular}

*Norma Técnica Servicios de Alimentación y Nutrición. Ministerio de Salud, 2005

Nutricionista Jefe del Servicio de Alimentación Colectiva, durante el año 2012, elaborado en base a los ítems de la "Norma Técnica para el Servicio de Alimentación y Nutrición" (MINSAL, marzo de 2005) e índice de nutricionistas por servicio clínico (Resolución Exenta N 209, del MINSAL, abril de 2005). El instrumento contenía preguntas sobre tipo de hospital, número total de camas y por servicio, número de raciones promedio diarias y número de nutricionistas por servicio de especialidad.

Posteriormente, el análisis de los datos fue realizado clasificando los hospitales públicos en forma dicotómica: 1) Grupo 1: hospitales públicos regionales y base y 2) Grupo 2: resto de los hospitales públicos. En el cálculo del requerimiento de nutricionistas se aplicó el criterio de la "Norma Técnica para Servicio de Alimentación y Nutrición", que califica los hospitales según nivel de complejidad y estima de acuerdo a ello, el número de nutricionistas por hospital (Tabla 1).

En los hospitales que no fue posible obtener el número de raciones, el cálculo se realizó en base al número de camas del hospital y del índice de nutricionistas por número de camas por servicio clínico (Tabla 2).

En el caso del Grupo 1, se utilizó la norma MINSAL 2005 y el índice de nutricionistas por servicio clínico, tal cual se indica en la tabla, sin embargo, en el caso del Grupo 2, se calculó el promedio de camas de los servicios para obtener un solo índice, el cual correspondió a 1 nutricionista por cada 31 camas, el que fue aplicado a este grupo.

Por lo tanto, el requerimiento total de nutricionistas consideró:

Grupo 1: Cantidad de nutricionistas, según nivel de complejidad norma MINSAL 2005 más cantidad de nutricionistas, según índice de nutricionistas por servicio clínico. 
Tabla 2. Índice nutricionista según servicio clínico*

\begin{tabular}{|ll|}
\hline Servicio & n de nutricionistas \\
\hline Medicina & 1 nutricionista por 20 camas \\
\hline Cirugía Adulto & 1 nutricionista por 22 camas \\
\hline Pediatría y Cirugía Infantil & 1 nutricionista por 29 camas \\
\hline Traumatología y Ortopedia & 1 nutricionista por 36 camas \\
\hline $\begin{array}{l}\text { Ginecología y Obstetricia } \\
\text { Especialidades, } \\
\text { pensionados y otros }\end{array}$ & 1 nutricionista por 36 camas \\
\hline
\end{tabular}

*Resolución Exenta № 209, de abril de 2005, Ministerio de Salud, Chile.

Grupo 2: Cantidad de nutricionistas, según nivel de complejidad norma MINSAL 2005 más cantidad de nutricionistas, según índice de nutricionista promedio (1 nutricionista por 31 ).

Finalmente, los datos recolectados de la dotación de nutricionistas 2012 de los hospitales públicos del estudio fueron comparados con el cálculo del requerimiento de nutricionistas, según los criterios mencionados anteriormente, para determinar el déficit existente.

En este estudio no se consideraron las raciones que se preparan en cada establecimiento para personal.

Norma Técnica del Servicio de Alimentación y Nutrición (MINSAL 2005). Regula la actividad, organización y el funcionamiento de los servicios de alimentación de los establecimientos de salud y unidades centrales de producción de alimentos. Define el "Servicio de Alimentación y Nutrición" como la organización que tiene como finalidad elaborar y/o distribuir alimentación científicamente planificada, de acuerdo a recomendaciones nutricionales nacionales e internacionales, a través de platos preparados u otras preparaciones culinarias, con estándares técnicos y sanitarios destinados a la alimentación de pacientes hospitalizados, pacientes ambulatorios, usuarios sanos o clientes en general.

Esta norma, además, aplica el "Reglamento Sanitario de los Alimentos" (DS: 977) vigente, el "Reglamento sobre Condiciones Sanitarias y Ambientales Básicas en los Lugares de Trabajo" (DS: 594) y ordenanza general de urbanismo y construcción. Se indica que todas las disposiciones contenidas en esta norma deben ser aplicadas ad integrum por los establecimientos nuevos de salud y a los existentes se les otorgará un plazo para normalizar sus instalaciones ${ }^{12-14}$.

La norma pone de relieve el rol de la alimentación y nutrición en el estado nutricional de los individuos sanos y el impacto que tiene en la recuperación de los pacientes hospitalizados, particularmente en pacientes críticos e inmuno-deprimidos, el que se refleja en el promedio de sus días de estada.

Asimismo, es clara al señalar que será responsabilidad del Servicio de Salud brindar una asistencia alimentaria nutricional a pacientes hospitalizados y usuarios que la dirección de establecimiento defina, respondiendo a las necesidades nutricionales a través de una alimentación técnicamente planificada y de óptimas condiciones sanitarias. Esta responsabilidad se encuentra estrictamente ligada al rol profesional del nutricionista y se materializa por medio de las funciones o tareas designadas por la ley de autoridad sanitaria (Ley 19.937 Art. $\left.4^{\circ}\right)^{15}$.

La norma define el número de nutricionistas según complejidad del establecimiento hospitalario (Tabla 1).

Los datos recolectados y calculados fueron tabulados en una plantilla del Programa Microsoft Excel 2010, expresando los datos en frecuencias absolutas y relativas, promedios, desviaciones estándar, mínimos y máximos.

\section{Resultados}

\section{Cobertura del recurso nutricionista por hospitales a nivel nacional}

La aplicación de las normativas indica que la dotación de nutricionistas a nivel nacional debería ser de 1.396 profesionales, en el año 2012. Se observó que se disponía de 603 nutricionistas (43\%), señalando un déficit de 794 profesionales (57\%) (Figura 1).

La cobertura por hospitales variaba de $11 \%$, en un hospital de máxima complejidad (Hospital Dr Philippe Pinel de Putaendo), a 120\% en un hospital de mínima complejidad (Hospital de Molina). Sólo en cinco hospitales de mínima complejidad (Molina, Teno, Lota, Traiguén y Calbuco) correspondiente a 2,9\% del estudio, se observaba la norma. Apenas 2,3\% estaba sobre los requerimientos y sólo $45,8 \%$ tenía una cobertura entre $50 \%$ y menos de $60 \%$ (Tabla 3 ). 


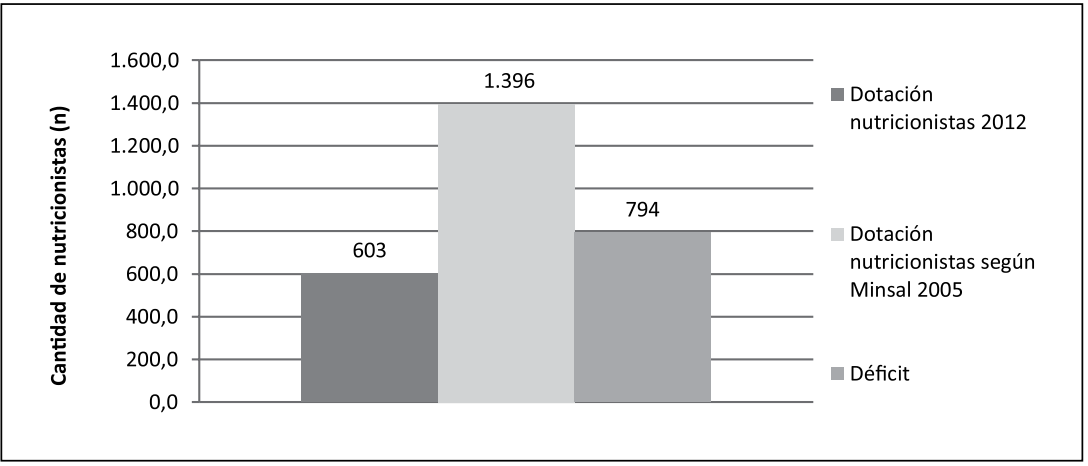

Figura 1. Dotación nutricionistas nivel nacional y déficit respecto a normativas Ministerio de Salud (*) Cantidad (número). Chile, 2012. *Ministerio de Salud. Norma Técnica Servicios de Alimentación y Nutrición. Santiago, Chile. 2005 y Resolución Exenta $N^{\circ} 209$, de abril de 2005, Santiago, Chile, 2005.

Tabla 3. Dotación nutricionistas 2012 por hospitales y regiones. Cobertura y déficit respecto a normas Ministerio de Salud*. Número y porcentaje (\%)

\begin{tabular}{|c|c|c|c|c|c|c|c|}
\hline \multirow[t]{2}{*}{ Ciudad } & \multirow[t]{2}{*}{ Hospital } & \multirow{2}{*}{$\begin{array}{l}\text { Comple- } \\
\text { jidad }\end{array}$} & \multirow{2}{*}{$\begin{array}{c}\text { Dotación } \\
\text { nutricionistas } \\
2012 \\
\text { n }\end{array}$} & \multirow{2}{*}{$\begin{array}{c}\text { Dotación } \\
\text { nutricionis- } \\
\text { tas }^{1,2} \\
\text { n }\end{array}$} & \multirow{2}{*}{$\begin{array}{c}\begin{array}{c}\text { Cober- } \\
\text { tura }\end{array} \\
\quad \%\end{array}$} & & icit \\
\hline & & & & & & $\mathbf{n}$ & $\%$ \\
\hline \multirow[t]{2}{*}{ Arica } & Regional de Arica & Máxima & 9 & 19 & 47,4 & 10 & 52,6 \\
\hline & Total & & 9 & 19 & 47,4 & 10 & 52,6 \\
\hline \multirow[t]{2}{*}{ Iquique } & Regional de Iquique & Máxima & 8 & 24 & 33,3 & 16 & 66,7 \\
\hline & Total & & 8 & 24 & 33,3 & 16 & 66,7 \\
\hline Antofagasta & Regional de Antofagasta & Máxima & 12 & 27 & 42,6 & 16 & 57,4 \\
\hline Calama & Hospital de Calama & Mínima & 3 & 4 & 75,0 & 1 & 25,0 \\
\hline Taltal & Hospital de Taltal & Mínima & 1 & 2 & 50,0 & 1 & 50,0 \\
\hline \multirow[t]{2}{*}{ Tocopilla } & Hospital de Tocopilla & Mínima & 2 & 5 & 40,0 & 3 & 60,0 \\
\hline & Total & & 18 & 38 & 46,1 & 21 & 53,9 \\
\hline Copiapó & Regional de Copiapó & Máxima & 7 & 19 & 36,8 & 12 & 63,2 \\
\hline Vallenar & Hospital de Vallenar & Mediana & 4 & 10 & 40,0 & 6 & 60,0 \\
\hline Chañaral & Hospital de Chañaral & Mínima & 1 & 2 & 50,0 & 1 & 50,0 \\
\hline Diego de Almagro & Hospital de Diego de Almagro & Mínima & 1 & 2 & 50,0 & 1 & 50,0 \\
\hline \multirow[t]{2}{*}{ Huasco } & Hospital de Huasco & Mínima & 1 & 2 & 50,0 & 1 & 50,0 \\
\hline & Total & & 14 & 35 & 40,0 & 21 & 60,0 \\
\hline La Serena & Hospital Regional de La Serena & Máxima & 6 & 18 & 33,3 & 12 & 66,7 \\
\hline Andacollo & Hospital de Andacollo & Mínima & 1 & 2 & 50,0 & 1 & 50,0 \\
\hline Combarbalá & Hospital de Combarbalá & Mínima & 1 & 2 & 50,0 & 1 & 50,0 \\
\hline Coquimbo & Hospital de Coquimbo & Máxima & 8 & 18 & 44,4 & 10 & 55,6 \\
\hline Illapel & Hospital de Illapel & Mínima & 1 & 3 & 33,3 & 2 & 66,7 \\
\hline Los Vilos & Hospital de Los Vilos & Mínima & 1 & 2 & 50,0 & 1 & 50,0 \\
\hline Ovalle & Hospital de Ovalle & Mediana & 6 & 11 & 54,5 & 5 & 45,5 \\
\hline \multirow[t]{2}{*}{ Vicuña } & Hospital de Vicuña & Mínima & 1 & 2 & 50,0 & 1 & 50,0 \\
\hline & Total & & 25 & 58 & 43,1 & 33 & 56,9 \\
\hline
\end{tabular}




\begin{tabular}{|c|c|c|c|c|c|c|c|}
\hline \multirow[t]{2}{*}{ Ciudad } & \multirow[t]{2}{*}{ Hospital } & \multirow{2}{*}{$\begin{array}{l}\text { Comple- } \\
\text { jidad }\end{array}$} & \multirow{2}{*}{$\begin{array}{c}\text { Dotación } \\
\text { nutricionistas } \\
2012 \\
\text { n }\end{array}$} & \multirow{2}{*}{$\begin{array}{l}\text { Dotación } \\
\text { nutricionis- } \\
\text { tas }^{1,2} \\
n\end{array}$} & \multirow{2}{*}{$\begin{array}{c}\begin{array}{c}\text { Cober- } \\
\text { tura }\end{array} \\
\%\end{array}$} & \multicolumn{2}{|c|}{ Déficit } \\
\hline & & & & & & $\mathbf{n}$ & $\%$ \\
\hline Valparaíso & Hospital Van Buren & Máxima & 11 & 28 & 39,3 & 17 & 60,7 \\
\hline Casablanca & Hospital de Casablanca & Mínima & 1 & 2 & 50,0 & 1 & 50,0 \\
\hline San Antonio & Hospital de San Antonio & Mínima & 3 & 4 & 75,0 & 1 & 25,0 \\
\hline Valparaíso & Hospital Eduardo Pereira & Mediana & 2 & 13 & 15,5 & 11 & 84,5 \\
\hline Valparaíso & Hospital del Salvador & Mínima & 1 & 3 & 30,7 & 2 & 69,3 \\
\hline Cabildo & Hospital de Cabildo & Mínima & 1 & 2 & 57,4 & 1 & 42,6 \\
\hline La Calera & Hospital de La Calera & Mínima & 1 & 3 & 28,7 & 2 & 71,3 \\
\hline La Ligua & Hospital de La Ligua & Mínima & 1 & 3 & 32,0 & 2 & 68,0 \\
\hline Limache & Hospital Geriátrico de Limache & Mínima & 1 & 6 & 18,1 & 5 & 81,9 \\
\hline Limache & Hospital de Limache & Mínima & 1 & 3 & 36,5 & 2 & 63,5 \\
\hline Petorca & Hospital de Petorca & Mínima & 1 & 2 & 50,0 & 1 & 50,0 \\
\hline Quillota & Hospital de Quillota & Mediana & 3 & 14 & 22,2 & 11 & 77,8 \\
\hline Quilpué & Hospital de Quilpué & Mediana & 3 & 10 & 29,5 & 7 & 70,5 \\
\hline Quintero & Hospital de Quintero & Mínima & 1 & 2 & 50,8 & 1 & 49,2 \\
\hline Villa Alemana & Hospital de Peñablanca & Mínima & 1 & 4 & 27,7 & 3 & 72,3 \\
\hline Viña del Mar & Hospital Gustavo Fricke & Máxima & 6 & 24 & 25,0 & 18 & 75,0 \\
\hline Llay - Llay & Hospital de Llay - Llay & Mínima & 1 & 2 & 50,8 & 1 & 49,2 \\
\hline Los Andes & Hospital de Los Andes & Mediana & 5 & 10 & 50,0 & 5 & 50,0 \\
\hline Putaendo & Hospital de Putaendo & Máxima & 2 & 18 & 11,1 & 16 & 88,9 \\
\hline Putaendo & $\begin{array}{l}\text { Hospital Psiquiátrico de } \\
\text { Putaendo }\end{array}$ & Mínima & 1 & 2 & 60,8 & 1 & 39,2 \\
\hline \multirow[t]{2}{*}{ San Felipe } & Hospital de San Felipe & Mediana & 5 & 13 & 37,5 & 8 & 62,5 \\
\hline & Total & & 52 & 167 & 31,1 & 115 & 68,9 \\
\hline Rancagua & Hospital Regional de Rancagua & Máxima & 8 & 28 & 28,6 & 20 & 71,4 \\
\hline Chimbarongo & Hospital de Chimbarongo & Mínima & 1 & 2 & 50,0 & 1 & 50,0 \\
\hline Coinco & Hospital de Coinco & Mínima & 1 & 2 & 50,0 & 1 & 50,0 \\
\hline Graneros & Hospital de Graneros & Mínima & 1 & 2 & 50,0 & 1 & 50,0 \\
\hline Litueche & Hospital de Litueche & Mínima & 1 & 2 & 50,0 & 1 & 50,0 \\
\hline Lolol & Hospital de Lolol & Mínima & 1 & 2 & 50,0 & 1 & 50,0 \\
\hline Marchigüe & Hospital de Marchigüe & Mínima & 1 & 2 & 50,0 & 1 & 50,0 \\
\hline Nancagua & Hospital de Nancagua & Mínima & 1 & 2 & 50,0 & 1 & 50,0 \\
\hline Peumo & Hospital de Peumo & Mínima & 1 & 3 & 33,3 & 2 & 66,7 \\
\hline Pichidegua & Hospital de Pichidegua & Mínima & 1 & 2 & 50,0 & 1 & 50,0 \\
\hline Pichilemu & Hospital de Pichilemu & Mínima & 1 & 2 & 50,0 & 1 & 50,0 \\
\hline Rengo & Hospital de Rengo & Mínima & 2 & 4 & 50,0 & 2 & 50,0 \\
\hline San Fernando & Hospital de San Fernando & Mediana & 4 & 11 & 36,4 & 7 & 63,6 \\
\hline San Vicente & Hospital de San Vicente & Mínima & 2 & 3 & 66,7 & 1 & 33,3 \\
\hline \multirow[t]{2}{*}{ Santa Cruz } & Hospital de Santa Cruz & Mínima & 2 & 4 & 50,0 & 2 & 50,0 \\
\hline & Total & & 28 & 71 & 39,4 & 43 & 60,6 \\
\hline
\end{tabular}




\begin{tabular}{|c|c|c|c|c|c|c|c|}
\hline Linares & Hospital Base de Linares & Máxima & 9 & 21 & 42,9 & 12 & 57,1 \\
\hline Talca & Hospital Regional de Talca & Máxima & 15 & 24 & 62,5 & 9 & 37,5 \\
\hline Cauquenes & Hospital de Cauquenes & Mínima & 2 & 5 & 40,0 & 3 & 60,0 \\
\hline Chanco & Hospital de Chanco & Mínima & 1 & 2 & 50,0 & 1 & 50,0 \\
\hline Constitución & Hospital de Constitución & Mínima & 3 & 3 & 100,0 & 0 & 0,0 \\
\hline Curepto & Hospital de Curepto & Mínima & 1 & 2 & 50,0 & 1 & 50,0 \\
\hline Curicó & Hospital de Curicó & Mediana & 10 & 13 & 76,9 & 3 & 23,1 \\
\hline Hualañe & Hospital de Hualañe & Mínima & 1 & 2 & 50,0 & 1 & 50,0 \\
\hline Licantén & Hospital de Licantén & Mínima & 1 & 2 & 50,0 & 1 & 50,0 \\
\hline Molina & Hospital de Molina & Mínima & 4 & 3 & 133,3 & -1 & $-33,3$ \\
\hline San Juan de la Costa & Hospital San Juan de la Costa & Mínima & 1 & 2 & 50,0 & 1 & 50,0 \\
\hline San Pablo & Hospital de Quilacahuín & Mínima & 1 & 2 & 50,0 & 1 & 50,0 \\
\hline Puerto Montt & $\begin{array}{l}\text { Hospital Regional de Puerto } \\
\text { Montt }\end{array}$ & Máxima & 12 & 25 & 48,0 & 13 & 52,0 \\
\hline Calbuco & Hospital de Calbuco & Mínima & 2 & 2 & 100,0 & 0 & 0,0 \\
\hline Castro & Hospital de Castro & Mínima & 6 & 5 & 120,0 & -1 & $-20,0$ \\
\hline Fresia & Hospital de Fresia & Mínima & 1 & 2 & 50,0 & 1 & 50,0 \\
\hline Frutillar & Hospital de Frutillar & Mínima & 1 & 2 & 50,0 & 1 & 50,0 \\
\hline Llanquihue & Hospital de Llanquihue & Mínima & 1 & 2 & 50,0 & 1 & 50,0 \\
\hline Maullín & Hospital de Maullín & Mínima & 1 & 2 & 50,0 & 1 & 50,0 \\
\hline Palena & Hospital de Palena & Mínima & 1 & 2 & 50,0 & 1 & 50,0 \\
\hline \multirow[t]{2}{*}{ Puerto Montt } & ELEAM & Mínima & 1 & 3 & 33,3 & 2 & 66,7 \\
\hline & Total & & 38 & 76 & 50,0 & 38 & 50,0 \\
\hline Coihaique & Hospital Regional de Coihaique & Mediana & 5 & 12 & 41,7 & 7 & 58,3 \\
\hline \multirow[t]{2}{*}{ Chile Chico } & Hospital de Chile Chico & Mínima & 1 & 2 & 50,0 & 1 & 50,0 \\
\hline & Total & & 6 & 14 & 42,9 & 8 & 57,1 \\
\hline Punta Arenas & $\begin{array}{l}\text { Hospital Regional de Punta } \\
\text { Arenas }\end{array}$ & Máxima & 9 & 22 & 40,9 & 13 & 59,1 \\
\hline Porvenir & Hospital de Porvenir & Mínima & 1 & 2 & 50,0 & 1 & 50,0 \\
\hline \multirow[t]{2}{*}{ Puerto Natales } & Hospital de Puerto Natales & Mínima & 1 & 2 & 50,0 & 1 & 50,0 \\
\hline & Total & & 11 & 26 & 42,3 & 15 & 57,7 \\
\hline
\end{tabular}

*Ministerio de Salud. Norma Técnica 2005. Servicios de Alimentación y Nutrición. Santiago, Chile, MINSAL 2015. *Resolución Exenta N²09, de abril de 2005, Santiago, Chile, MINSAL 2015.

\section{Cobertura del recurso nutricionista por regiones}

En regiones la cobertura del recurso nutricionista variaba de 27,3\% en la Región de Valparaíso a 62,3\% en la Región del Maule, siendo esta última la única en esa condición. Cabe destacar que sólo 3 regiones presentaban una cobertura entre 50\% y $55 \%$, equivalente al $5 \%$. El déficit promedio de cobertura por total regiones es de 57\% (Tabla 4).

\section{Cobertura del recurso nutricionista según complejidad de los hospitales}

Máxima, mediana y mínima complejidad. El análisis según nivel de complejidad señala que la cobertura variaba entre $43 \%$, en los de mínima complejidad, que en su mayoría correspondía a una dotación única de 1 profesional, a $57 \%$, en los de mediana complejidad. Destaca el déficit 
Tabla 4. Dotación de nutricionistas según regiones 2012. Cobertura y déficit respecto a normas de Ministerio de Salud (*) Cantidad (Número) y porcentaje (\%). Chile, 2012

\begin{tabular}{|c|c|c|c|c|c|}
\hline \multirow[t]{2}{*}{ Región } & \multirow{2}{*}{$\begin{array}{c}\text { Dotación } \\
\text { nutricionistas } \\
2012\end{array}$} & \multirow{2}{*}{$\begin{array}{l}\text { Dotación nutricionistas } \\
\text { según norma } \\
\text { MINSAL } 2005\end{array}$} & \multirow{2}{*}{$\begin{array}{c}\text { Cobertura } \\
\%\end{array}$} & \multicolumn{2}{|c|}{ Déficit } \\
\hline & & & & $\mathbf{n}$ & $\%$ \\
\hline Arica & 9,0 & 19,2 & 47,0 & 10,2 & 53,0 \\
\hline Tarapacá & 10,0 & 24,0 & 41,7 & 14,0 & 58,3 \\
\hline Antofagasta & 17,5 & 39,4 & 44,4 & 21,9 & 55,6 \\
\hline Atacama & 14,0 & 34,6 & 40,4 & 20,6 & 59,6 \\
\hline Coquimbo & 26,0 & 59,1 & 44,0 & 33,1 & 56,0 \\
\hline Valparaíso & 51,0 & 186,5 & 27,3 & 135,5 & 72,7 \\
\hline O`Higgins & 28,0 & 67,0 & 41,8 & 39,0 & 58,2 \\
\hline Maule & 54,0 & 86,6 & 62,3 & 32,6 & 37,7 \\
\hline Biobío & 98,0 & 194,3 & 50,4 & 96,3 & 49,6 \\
\hline Araucanía & 46,0 & 99,0 & 46,5 & 53,0 & 53,5 \\
\hline Los Ríos & 17,0 & 41,6 & 40,8 & 24,6 & 59,2 \\
\hline Los Lagos & 44,0 & 87,8 & 50,1 & 44 & 49,9 \\
\hline Aysén & 10,0 & 18,3 & 54,7 & 8,3 & 45,3 \\
\hline Magallanes & 11,0 & 26,2 & 41,9 & 15,2 & 58,1 \\
\hline Metropolitana & 167,0 & 412,7 & 40,5 & 245,7 & 59,5 \\
\hline Total & 602,5 & $1.396,3$ & 43,0 & 794,0 & 57,0 \\
\hline
\end{tabular}

*Ministerio de Salud. Norma Técnica 2005. Servicios de Alimentación y Nutrición. Santiago, Chile, MINSAL 2015. *Resolución Exenta N²09, de abril de 2005, Santiago, Chile, MINSAL 2015.

de $46 \%$ en los hospitales de máxima complejidad (969 profesionales) (Figuras 2, 3, 4).

\section{Discusión}

El recurso nutricionista en los hospitales públicos estudiados presenta un déficit significativo respecto a las normativas vigentes, que señalan que debería ser de 1.396 profesionales, logrando una cobertura sólo de $43 \%$, con 602 nutricionistas y un déficit de $60 \%$, de 794 profesionales. Esta situación no permite responder al objetivo principal del profesional disciplinario "Optimizar la alimentación y nutrición que se otorga en establecimientos asistenciales $\mathrm{u}$ otras instituciones públicas o privadas, a fin de contribuir eficientemente en la mantención o recuperación de la salud de los individuos" 10 .

Teniendo presente que la nutrición clínica aborda el estado nutricional de las personas como el resultado de la interrelación de múltiples factores biológicos, psicológicos y sociales, desde la prevención de los problemas nutricionales más frecuentes, al tratamiento de la enfermedad y sus complicaciones, para lo cual es imprescindible la utilización de herramientas diagnósticas, como la valoración nutricional, encuestas dietéticas, antropometría, indicadores bioquímicos e intervenciones terapéuticas específicas para entregar una alimentación oral y el desarrollo de la asistencia nutricional intensiva (CEFE) a los usuarios hospitalizados, mejorando su calidad de vida. La desnutrición hospitalaria en Latinoamérica se presenta como un grave problema y pocos países cuentan con políticas que normalicen estas prácticas ${ }^{16-19}$.

Según la declaración de Cancún, emitida por los presidentes de las sociedades y asociaciones de Nutrición Clínica y Terapia Nutricional de América Latina y el Caribe, en el marco del XI 

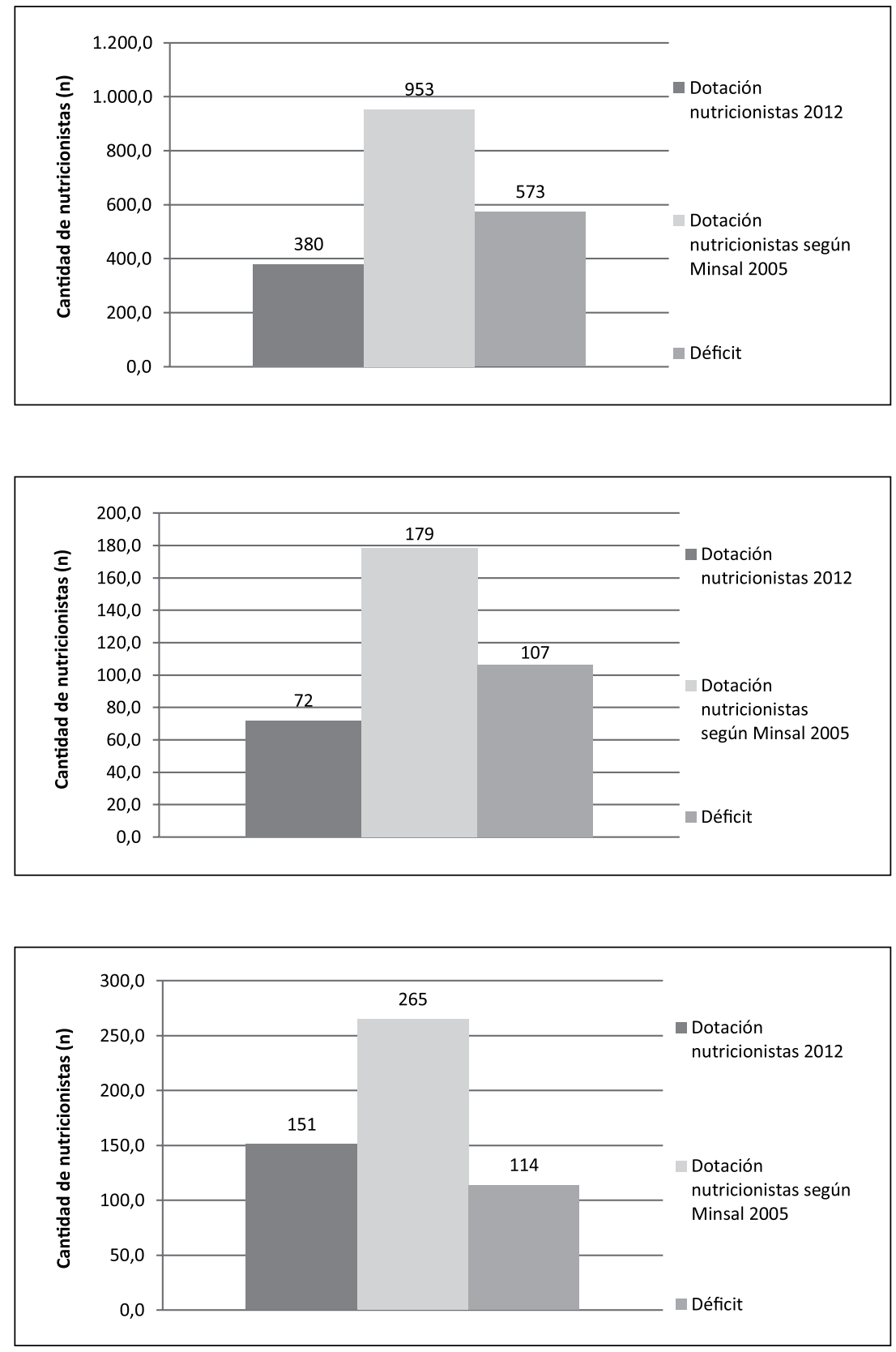

Figura 2. Dotación nutricionista hospitales máxima complejidad comparada con normativa Ministerio de Salud $\left.{ }^{*}\right)$ y déficit. Cantidad (número), Chile, 2012. *Ministerio de Salud. Norma Técnica Servicios de Alimentación y Nutrición. Santiago, Chile. 2005 y Resolución Exenta $N^{\circ} 209$, de abril de 2005, Santiago, Chile, 2005.

Figura 3. Dotación nutricionista hospitales mediana complejidad a nivel nacional comparada con normativas Ministerio de Salud* y déficit. Cantidad (número), Chile, 2012. *Ministerio de Salud. Norma Técnica Servicios de Alimentación y Nutrición. Santiago, Chile, 2005 y Resolución Exenta N²09, de abril de 2005, Santiago, Chile, MINSAL 2015.

Figura 4. Dotación nutricionista hospitales baja complejidad a nivel nacional comparada con normativas Ministerio de Salud* y déficit. Cantidad (número), Chile, 2012. *Ministerio de Salud. Norma Técnica Servicios de Alimentación y Nutrición. Santiago, Chile, 2005 y Resolución Exenta $N^{\circ} 209$, de abril de 2005, Santiago, Chile, MINSAL 2015.
Congreso de FELANPE/XV Congreso de la Asociación Mexicana de Nutrición Clínica y Terapia Nutricional (AMAEE), en la ciudad de Cancún, Quintana Roo, México, el día 20 de mayo de 2008 y el documento de consenso de funciones y competencias del nutricionista clínico, en el marco del XIII Congreso de FELANPE 2012, realizado en Panamá, señalan que los enfermos tienen: i) el derecho humano a recibir una terapia nutricional oportuna y óptima en calidad y cantidad, en cualquier lugar donde se encuentren; ii) Respecto a la evaluación y tratamientos nutricionales en los hospitales, se acuerda que la atención nutricional es una necesidad impostergable para todo paciente; 
iii) El soporte nutricional es obligatorio para todo paciente malnutrido o en riesgo. Además hay que considerar los costos económicos (costo/beneficio) que implica que un paciente de alta complejidad, por las patologías que presente, no reciba la atención especializada, aumentando los días de estadía y la ausencia de atención en las unidades de servicios intensivos (UCI), medicina, pacientes con complicaciones renales y metabólicas ${ }^{20,21}$.

La situación de déficit del nutricionistas se presenta en todo el país, en menor o mayor intensidad. Esta situación se arrastra desde la segunda mitad de la década 1970-79, cuando las nuevas orientaciones de las políticas de salud que se implementaron, plantearon que los nutricionistas clínicos de los hospitales públicos fueran llevados a la atención primaria, dejando una mínima dotación de nutricionistas en éstos, desarticulando el trabajo técnico que se realizaba en nutrición clínica, sin que se haya logrado recuperar los cargos clínicos y revertir la situación generada, hasta la actualidad.

\section{Recomendaciones}

Desde esta perspectiva, es importante que desde el Estado y el MINSAL se realicen acciones de política tendientes a reponer el recurso nutricionista en todas las áreas del ejercicio profesional clínico hospitalario. La evidencia de estudios internacionales señalan la importancia de la dietoterapia y el control nutricional de los pacientes hospitalizados, por una parte los altos índices de desnutrición a nivel clínico, y por los requerimientos de la dietoterapia cada vez más especializada por la complejidad de las enfermedades, lo que traducirá en el análisis del costo/beneficio asociado a la recuperación de los pacientes con menos días de estadía, menores complicaciones y mejor respuesta a los tratamientos ${ }^{7,8,16,17}$. Los antecedentes expuestos ponen de manifiesto la precariedad del recurso nutricionista y denota, a la vez, una ausencia o atomización de la política en recursos humanos, desde los niveles centrales y regionales, para el abordaje de los problemas de salud y necesidades de recursos profesionales.

Como señala la OMS, los recursos humanos conforman la base social y técnica de los sistemas de salud, lo que implica una responsabilidad social de gran importancia para el desarrollo humano, lo que contribuye a lograr un equilibrio entre los derechos y responsabilidades sociales de los trabajadores de la salud y los ciudadanos que merecen la atención y el derecho a la salud ${ }^{22-24}$.

Este estudio tiene una connotación social, ya que aporta antecedentes para orientar políticas de recursos humanos de nutricionistas en el nivel secundario de salud en Chile y puede contribuir a lograr los propósitos de la OPS “de promover acciones y coordinar los esfuerzos de los países de la región para mejorar la salud y las condiciones de vida de los pueblos de las Américas ${ }^{5}$.

Agradecimientos: A los nutricionistas jefes del Servicio de Alimentación y Nutrición de los hospitales públicos que respondieron a la solicitud de completar el cuestionario. Al Colegio Nacional de Nutricionistas Universitarios de Chile por facilitar antecedentes de la Región Metropolitana. A Sofía Coñuecar, nutricionista que ordenó la base de datos y Lina Besaccia, Bibliotecóloga del Centro de Estudios Avanzados (CEA), UPLA.

\section{Referencias}

1. Ministerio de Salud, (MINSAL). Plan nacional de salud, objetivos sanitarios de la década 2011-2020. Estrategia nacional de salud 2011-2020; [Consultado el 3 de marzo de 2014].

2. Organización Naciones Unidas (ONU). Objetivos de Desarrollo del Milenio. Nueva York: ODM; 2003.

3. Organización Panamericana de la Salud (OPS), Ministerio de Salud de Ontario, Canadá. Llamado a la acción de Toronto, Hacia una década de recursos humanos para la salud en las Américas. Toronto, Canadá: OPS; 2005.

4. Organización Panamericana de Salud-Organización Mundial de la Salud, (OPS/OMS). Manual de medición y monitoreo indicadores de las metas regionales de recursos humanos para la salud. Un compromiso compartido OPS/OMS. Washington, DC USA, OPS/OMS; 2011.

5. Organización Mundial de la Salud (OMS). Agenda de Salud para las Américas 2008-2017, Agenda presentada por los ministros de salud de las Américas; 2007 [Consultado el 3 de junio de 2013]. Disponible en: http:// www.paho.org/hq/index.php?option=com_content\&view=article\&id=1976\&Itemid $=1724$.

6. Ministerio de Salud (MINSAL). Encuesta Nacional de Salud: Informe preliminar. Santiago, Chile: MINSAL; 2009-2010.

7. Uauy R, Vio F. Health and nutrition transition in de- 
veloping countries: the case of Chile. En: The Nation's Nutrition. Washington, DC. USA: International Life Sciences; 2007. p. 117-28.

8. Ministerio de Salud, (MINSAL). Estudio de Prioridades de Inversión en Salud. Estudio Carga de Enfermedad. Informe Final. Santiago, Chile: MINSAL; 1996.

9. Ministerio de Salud. Informe final: Estudio de carga de enfermedad y carga atribuible. Santiago, Chile: MINSAL; 2008.

10. Ministerio de Salud, (MINSAL). Norma Técnica de Servicios de Alimentación y Nutrición. Santiago, Chile: MINSAL; 2005.

11. Ministerio de Salud, (MINSAL). Resolución Exenta $\mathrm{N}^{\circ}$ 209, de abril de 2005. Santiago, Chile, MINSAL; 2005.

12. Reglamento Sanitario de los Alimentos. Decreto Supremo 977/96. Modificado, Decreto 115/2003. Santiago, Chile: Diario Oficial 2003.

13. Condiciones Sanitarias y Ambientes Básicos de los Lugares de Trabajo. Decreto 594/2000. Santiago, Chile: Diario Oficial 2000.

14. Ministerio de Vivienda y Urbanismo. Ordenanza General de la Ley General de Urbanismo y Construcciones. Reglamento de Ley 19.537. Decreto supremo 46 de 1998. Modificado Decreto supremo 67/2014. Santiago de Chile. Diario Oficial 2004.

15. Ministerio de Salud. Ley de Autoridad Sanitaria. Ley No 19.937. Santiago de Chile, Diario Oficial 24.02.04.

16. Correia M, Campos A. Prevalence of hospital malnutrition in Latin America: the Multicenter ELAN Study. Nutrition 2003; 19: 823-5.
17. Labraña A, Durán E, Asenjo G, Hansel G. Plan de estudios basado en competencias para la carrera de Nutrición y Dietética de la Universidad de Concepción. Rev Chil Nutr 2010; 37 (3): 302-7.

18. Santana Porbén S. For the Cuban Group for the Study of Hospital Malnutrition. The state of provision of nutritional care to hospitalized patients. Results from the ELAN-CUBA Study. Clinical Nutrition 2006; 25: 1015-29.

19. Waitzberg DL, Ravacci GR, Raslan M. Desnutrición hospitalaria. Nutr Hosp 2011; 26 (2): 254-64.

20. Federación Latinoamericana de Terapia Nutricional, Nutrición Clínica y Metabolismo, (FELANPE). Declaración internacional de sobre el derecho a la nutrición en los hospitales; 2008 [Consultado el 31 de julio de 2014]. Disponible en: http://www.felanpeweb.org/dec_cancun_2008.pdf

21. Federación Latinoamericana de Terapia Nutricional, Nutrición Clínica y Metabolismo, (FELANPE). Documento de consenso funciones y competencias del nutricionista clínico; 2013.

22. Organización Mundial de la Salud Informe sobre la salud en el mundo 2003. Forjemos el futuro. OMS; 2003.

23. Organización Mundial de la Salud, (OMS). Informe sobre la salud en el mundo 2006. Colaboremos por la salud. OMS; 2006.

24. Organización Mundial de la Salud. Informe sobre la salud en el mundo 2013. Investigaciones para una cobertura sanitaria universal. Colaboremos por la salud. OMS; 2013. 